\title{
The REINFORCE citizen-science project and the search for new long-lived particles at the LHC
}

\section{S. Angelidakis", on behalf of the REINFORCE consortium}

National and Kapodistrian University of Athens, University Campus Zografou, Athens, Greece

E-mail: stylianos.angelidakis@cern.ch

The REINFORCE project engages and supports citizens to cooperate with researchers and actively contribute to the development of new knowledge for the needs of science and society. The aim is to bridge the gap between them, and reinforce society's science capital. Within this context, the demonstrator "New Particle Search at CERN" will engage citizen-scientists in searches for new elementary particles in the high-energy proton-proton collisions at the LHC. To make this possible, the demonstrator adopts a three-stage architecture. The first two stages use simulated data from the ATLAS detector to train citizens, but also to allow for a quantitative assessment of their performance and comparison with machine learning algorithms. The third stage uses real data from the ATLAS Open-Data subset, providing two research paths: (a) study of Higgs boson decays to two photons, one of which could be converted to an electron-positron pair, and (b) search for yet undiscovered long-lived particles predicted by certain theories Beyond the Standard Model.

The European Physical Society Conference on High Energy Physics (EPS_HEP2021) 26-30 July 2021

Online conference, jointly organized by Universität Hamburg and the research center DESY

"Speaker 


\section{Introduction}

REINFORCE [1] (Research Infrastructures FOR Citizens in Europe) is a Research \& Innovation Project, supported by the European Union's Horizon 2020 SWAFS "Science with and for Society" work programme. The project will create a series of cutting-edge projects on frontier Physics research, with citizen scientists making a genuine and valued contribution to managing the data avalanche.

More than 100,000 citizens will be engaged in research done in Large Research Infrastructures through a participatory design methodology that takes into account the special characteristics of different target groups, their barriers and constraints, their perceptions and biases and their attitudes and knowledge regarding science. Through the use and analysis of open data, citizens will perform their own inquiries, supported by the REINFORCE experts.

\section{The REINFORCE Demonstrators}

The Large Scale Citizen-Science demonstrators are the key vehicle that REINFORCE utilizes in order to bring frontier science and society together. They tackle different challenges in the fields of frontier Physics, including activities at the interplay of frontier science and environmental awareness. Each of them provides a design to data acquisition, data selection and monitoring the quality of citizens' input. All the demonstrators are developed by Large Research Infrastructures and are built on top of the infrastructures provided by the Zooniverse platform [2] utilizing its tools and technologies.

The role of citizens is crucial in each demonstrator: citizens are engaged in authentic research experiences, being asked for support in exploring uncharted scientific territories, taking tasks which are essential for the needs of scientific research. They are also provided with cut tailored educational resources and training material, and with open data that they may be able to use in order to inquire further with the guidance of the consortium experts. For this reason, the demonstrators are in constant development considering citizen feedback and recommendations. The four demonstrators that are developed to engage citizens are the following:

- Gravitational-Wave noise hunting: Its aim is to develop a cutting-edge citizen science programme by providing public access to gravitational-wave antenna data, including environmental data, for an open-data project.

- Deep Sea Hunters: Invites citizens to optimize the KM3NeT neutrino telescope against sources of environmental noise which have never been systematically studied, while engaging in the exciting world of neutrino astronomy.

- New Particle Search at CERN: Engages citizens in the quest of the LHC for the discovery of the physics beyond the Standard Model.

- Cosmic Muon Images: Interdisciplinary studies with geoscience and archaeology has the goal to show how the technology developed to study fundamental physics can be applied for the development of frameworks with significant impact on society.

In addition to the above, an important objective of REINFORCE is to extend the senses used in the scientific inference, beyond this of the visual, and include in the overall effort and scientific community sense-disabled people (especially visually-disabled) and senior citizens. 


\section{New Particle Search at CERN}

The Large Hadron Collider (LHC) at CERN is the world's most powerful particle accelerator. In its two $27 \mathrm{~km}$-long circular vacuum tubes, proton beams are accelerated to almost the speed of light, in opposite directions. The tubes intersect at four Interaction Points (IPs) along the LHC circumference, where the beams cross and protons collide. To study the collision products, four large experiments (particle detectors) are installed around the IPs. The largest, general-purpose detector is ATLAS [3], measuring $44 \mathrm{~m}$ long and $25 \mathrm{~m}$ high, and weighing over 7000 tons.

In 2012, ATLAS and CMS (the other general-purpose LHC experiment) co-discovered the Higgs boson [4][5], the last elementary constituent missing from our present description of nature and its laws, the Standard Model (SM) [6]. Despite this great success of the SM, open questions remain and there are several theoretical models Beyond the Standard Model (BSM) that try to answer them. Certain models predict the existence of Long-Lived Particles (LLPs) with lifetimes of the order of picoseconds to nanoseconds [7], which decay to known SM particles several millimetres or centimetres away from the IP at the centre of the detector. Due to their significant distance from the IP, such decay points are called Displaced Vertices (DVs) and their detection is important for research carried out in both the ATLAS and CMS experiments.

Based on the above, the goal of the demonstrator is to actively engage non-expert citizens in searches for new elementary particles that may be produced in the high-energy proton-proton collisions at the LHC (CERN). This is achieved through a three-stage process, which is described below along with the analysis tools developed for each of the stages.

\section{Stage 1 - Displaced vertex identification}

In the first stage of the demonstrator, citizen-scientists will inspect stationary images displaying reconstructed tracks of charged particles in the inner detector of ATLAS, such as the one shown in Figure 1. The candidate tracks are obtained from the set of tracks that have been reconstructed in the inner detector, satisfying the quality criteria used in searches of ATLAS for LLPs as well as few additional requirements to facilitate the identification by human eye.

Citizen-scientists have to identify the coordinates of any DV in each event, using their mouse pointer. All tracks associated with a primary vertex (i.e. a vertex close to the beam collision point) have been removed from the display so that a DV candidate may be identified as the common origin of any two or more tracks in the ATLAS inner detector. To facilitate the citizens' task, a detailed tutorial and a help section (including video instructions and several examples) is provided. The citizens' selection is internally assessed by Zooniverse based on the true location of the DV, which is also provided to the platform. Zooniverse presents feedback to the citizens after each processed event, before progressing to the next event.

In the ATLAS experiment, a variety of algorithms have been developed to automate the task of identifying DVs with varying degrees of accuracy depending on a large number of factors. As this is a task that, under certain conditions, can be easily performed by humans, the goal is to compare the accuracy and efficiency of an automated identification algorithm to that of human volunteers. Therefore, this stage makes use of simulated events, since they allow 


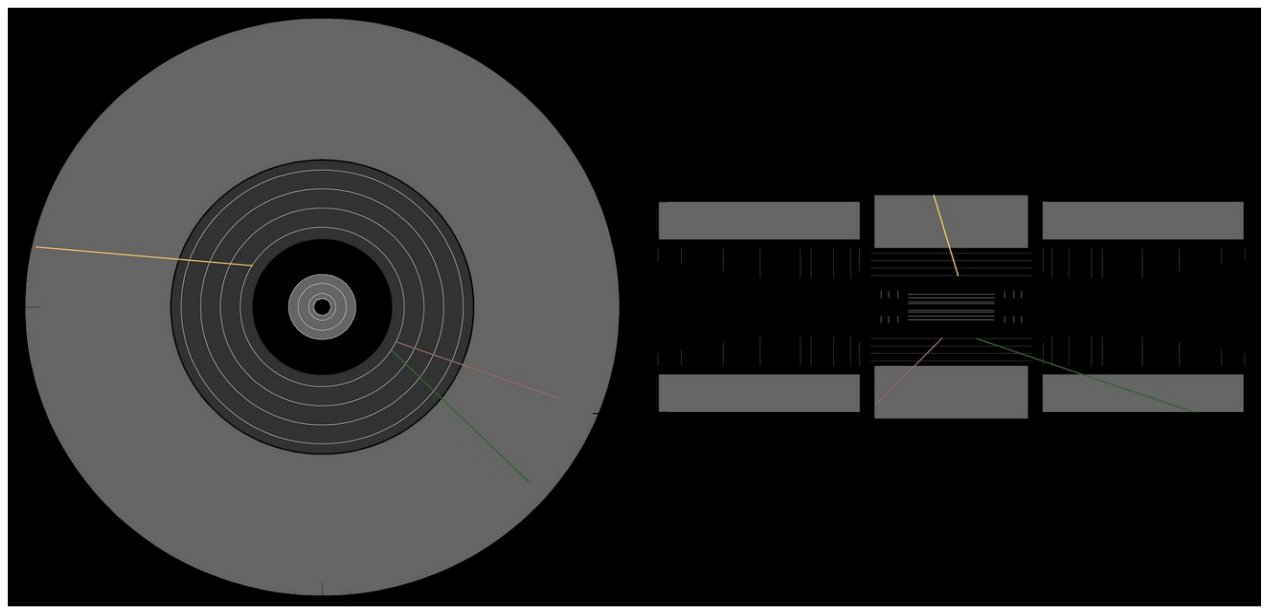

Figure 1: Collision event containing a DV, as displayed on Zooniverse. Two projections of the ATLAS inner detector are shown; transverse (left) and longitudinal (right).

knowing with absolute certainty the true position of each DV. The same events will be analysed by both the algorithm and citizen-scientists allowing for an accurate and impartial comparison.

\section{Stage 2 - Particle Identification}

In the second stage of the demonstrator, citizens-scientists go a step further; instead of examining stationary images of collision events, they interact with the event display provided by HYPATIA [8] (HYbrid Pupil's Analysis Tool for Interactions in ATLAS) to perform in-depth analysis and familiarize themselves with the identification of particles through their characteristic signatures. In each displayed event (Figure 2), citizen-scientists need to select individual tracks, in the ATLAS inner detector, or energy clusters, in the electromagnetic calorimeter, and classify them as muons, electrons, converted or non-converted photons using the functionalities of HYPATIA. For each selected track or cluster, they need to inspect its properties (momentum, charge, direction) to assist them in their classification.

The simulated event sample used for this stage is derived from the ATLAS Open Data set and includes: (a) Higgs bosons decaying to a pair of $Z$ bosons, leading into final states with four leptons (electrons or muons), which citizens are asked to identify, and (b) Higgs bosons decaying to a pair of photons, one of which may have been converted to e+e- pair. The use of simulated events is necessary to provide the true type of each particle, based on which, citizens' selections will be assessed.

As in Stage 1, citizen-scientist are provided with an extensive help section, with a description of the ATLAS detector and instructions on how to recognize each particle type, as well as a tutorial with examples for each type of particle. Their selections are automatically logged by HYPATIA for further analysis and feedback. Finally, the citizens' identification efficiency will be compared to that of a machine-learning algorithm using the same information in order for the comparison to be impartial.

\section{Stage 3 - Introduction of real data analysis tools}

In Stage 3, citizen-scientists will study real events recorded by the ATLAS detector during the second running period of the LHC, which are part of the $13 \mathrm{TeV}$ Open Data released by the 

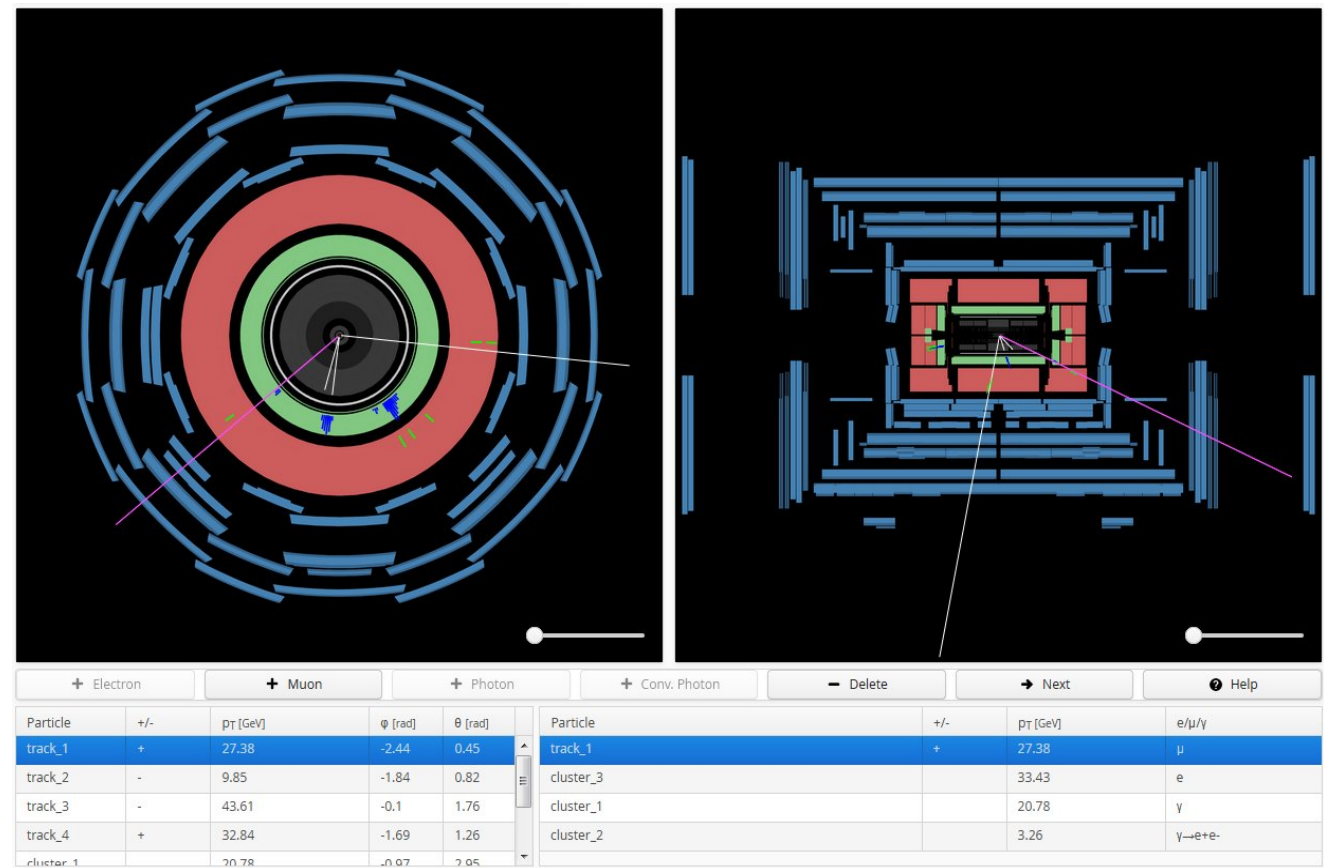

Figure 2: A collision event displayed by HYPATIA in Stage 2. Two projections of the entire ATLAS detector are shown; transverse (left) and longitudinal (right).

ATLAS collaboration. The stage is divided into two sub-stages: Stage 3a, titled "Study of Higgs Bosons", in which citizen-scientists will study the decays of Higgs bosons to photons, and Stage $3 b$, titled "Discovery of Long-Lived Particles", in which citizen-scientists have to select candidate events that may include the production and decay of a hypothesised LLP.

In both parts of Stage 3, citizen-scientists will interact more with the event display of HYPATIA, selecting traces of particles but also calculating kinematic quantities, such as the mass of Higgs boson, or possibly, of the sought-after LLP. For this purpose, HYPATIA has also been adapted to provide the necessary information and functionalities needed for each substage. As in previous stages, a detailed help section and brief tutorial are also provided.

\subsection{Stage 3a - Study of Higgs Bosons}

In Stage 3a, citizens-scientists are asked to identify energy clusters in the electromagnetic calorimeter of ATLAS, corresponding to unconverted or converted photons that may originate from the decay of a Higgs boson. In this task, HYPATIA assists by calculating the invariant mass of each pair of selected photons. In addition to photon selection, citizen-scientists are also provided with the functionality to rate each event, using a star rating system, according to its complexity and its probability to include a rare Higgs production mode.

Citizen-scientists' selections and event ratings are recorded by HYPATIA. At the end of the project, statistical analysis of the invariant mass histograms will follow in order to look for differences between three event cases: events with two unconverted photons, events with an unconverted and a converted photon and events with two converted photons. Finally, discussion on cases in which citizens have shown particular interest (through the star rating system) can follow in the project's discussion board. 


\subsection{Stage 3b - Discovery of Long-Lived Particles}

In Stage 3b, citizen scientists will be searching for LLP through the identification of DVs. After identifying a DV, they will proceed to select muons originating it and study their kinematic properties, as well as properties of the DV itself (e.g. its invariant mass and transverse distance from the beam-collision point) provided by HYPATIA. Furthermore, they are asked to rate each event, using the star-rating system, according to how consistent they believe it is with the production and decay of an LLP. This way, the citizens will be directly involved in potential discoveries without requiring any high level physics or computing skills.

As in Stage 3a, citizen-scientists' selections and event ratings are recorded by HYPATIA. At the end of the project, statistical analysis of the kinematic quantities populated with the measurements of all of the participating citizens will be performed in order to identify any evidence of the existence of LLPs. Discussion on cases in which citizens have shown particular interest (through the star rating system) can also follow in the project's discussion board.

\section{Conclusions}

Within the REINFORCE project, four demonstrators have been developed by Large Research Infrastructures to engage citizens in public-funded frontier research. All demonstrators are hosted by Zooniverse, which presently has over two million active users, and is therefore expected to bring each demonstrator to a wide range of citizens. The project will be made available to the public by Fall 2021.

\section{References}

[1] https://www.reinforceeu.eu.

[2] https://www.zooniverse.org.

[3] ATLAS Collaboration, The ATLAS Experiment at the CERN Large Hadron Collider, JINST 3 (2008) S08003.

[4] ATLAS Collaboration, Observation of a new particle in the search for the Standard Model Higgs boson with the ATLAS detector at the LHC, Phys. Lett. B 7161 (2012) 1-29.

[5] CMS Collaboration, Observation of a new boson at a mass of $125 \mathrm{GeV}$ with the CMS experiment at the LHC, Phys. Lett. B 7161 (2012) 30-61.

[6] S. Weinberg, The Making of the standard model, Eur. Phys. J. C34 (2004) 5-13.

[7] ATLAS Collaboration, Search for long-lived massive particles in events with a displaced vertex and a muon with large impact parameter in pp collisions at $\sqrt{s}_{s}=13 \mathrm{TeV}$, Phys. Rev. D 102 (2020) 032006 .

[8] C. Kourkoumelis C and S. Vourakis, HYPATIA - An online tool for ATLAS event visualization, Phys. Educ. 49 (2014) 21-32.

\section{Acknowledgements}

The REINFORCE project has received funding from the European Union's Horizon 2020 project call H2020-SwafS-2018-2020 funded project Grant Agreement no. 872859. 